\title{
Attentional selection of a peripheral ring overrules the central attentional bias
}

\author{
KARINA J. LINNELL and GLYN W. HUMPHREYS \\ University of Birmingham, Birmingham, England
}

\begin{abstract}
In visual search, distractors that fall between fixation and the target are more disruptive than distractors that fall at the same eccentricity as the target (Wolfe, O'Neill, \& Bennett, 1998). This sensitivity to the relative eccentricity of target and distractor elements originates from a space-based bias favoring stimuli closer to the fovea. We show that this spatial bias can be overruled by cuing attention to a ringshaped object. We rule out various space-based explanations of these findings, including (1) attention to fronto-parallel planes in depth and (2) serial attention to different portions of a ring. We suggest that attentional selection of a ring-shaped object operates independently of, and can overrule, spatial biases in selection.
\end{abstract}

Visual selection is subject to various forms of bias, one of which is a bias to favor stimuli near fixation over stimuli far from fixation. In visual search, for instance, targets at peripheral locations are detected more slowly than are targets at central locations (e.g., Carrasco, Evert, Chang, \& Katz, 1995). This advantage for central targets is not due to the differential involvement of eye movements for targets close to and far from fixation, since it occurs with display durations too brief for eye movements (Carrasco et al., 1995). Furthermore, although differences between peripheral and central targets can be reduced when stimuli are scaled for cortical magnification (Carrasco \& Frieder, 1997; Virsu \& Rovamo, 1979), a “central” advantage can still be found if, on peripheral target trials, distractors that share response-relevant features fall in more central regions.

Wolfe, O'Neill, and Bennett (1998) required participants to search for a target element defined by a conjunction of form and color features among distractor elements carrying either the target form or the target color. The relative eccentricities of target and distractor elements were manipulated, with cortical magnification controlled. Specifically, the target element could fall at different distances from fixation, and distractors could fall either all at the same eccentricity as the target or scattered across different eccentricities. In the latter case, as target eccentricity increased, so too did the number of distractors that fell closer to fixation than the target. Only in this case did search reaction times (RTs) increase with the target's ec-

This research was supported by grants from the Medical Research Council, U.K. Part of it was reported at the 23rd meeting of the European Conference of Visual Perception, August 2000. It has benefited from comments by J. M. Wolfe, C.N.L. Olivers, and two anonymous reviewers. Correspondence concerning this article should be addressed to K. J. Linnell at Department of Psychology, Goldsmiths College, University of London, London SE14 6NW, England (e-mail: k.j.linnell@gold.ac.uk).

Note-This article was accepted by the previous editorial team, headed by Neil Macmillan. centricity. Wolfe et al. attributed this effect of eccentricity to a bias to allocate attention toward more centrally presented elements. Thus, as the target moves into the periphery and increasing numbers of distractors fall to the center of the target, it wins an increasingly small share of attentional resources and is detected more and more slowly.

The bias favoring central over peripheral stimuli is consistent with space-based models of visual selection. For example, LaBerge and Brown (1989) assume that selection stems from the allocation of attention to a contiguous region of space around fixation in which the "attentional weight" attached to stimuli varies inversely with the distance of the stimuli from fixation. Thus, the bias is evident when there are multiple items present at different eccentricities, when the competition for selection depends on the relative amount of attentional weight available to stimuli at different eccentricities (see Bundesen, 1990, for a detailed account). When single items are presented, or multiple items at a single eccentricity, the bias should have little effect.

In addition to space-based accounts of selection, other evidence indicates that participants can attend selectively to elements falling in regions of space that group together to form an object (Duncan, 1984; Vecera \& Farah, 1994). Indeed, elements falling in an overlapping region of space that is not part of the target object may not be attended, even if they are equidistant from fixation and thus equally likely to attract attention on a space-based view (Duncan, 1984). A distinction has been drawn in the literature between attention to objects abstracted from their locations and attention to grouped locations (see Vecera \& Farah, 1994). We will not seek to discriminate between these accounts, either of which could be applied to the results we present. For simplicity, we will use the term object-based attention, but the reader should bear in mind that the "objectbased" selection effects we refer to below could come about through selecting the grouped locations that objects occupy. 
Object-based attention can be put into direct opposition with space-based attention by using ring-shaped objects, which are important stimuli for evaluating the relations between object- and space-based attention because they arise from grouping across noncontiguous spatial regions in the periphery. In this article, we ask whether attention to noncontiguous ring-shaped objects is possible by pitting selection of a ring of peripheral elements against a default spatial bias that we show favors central elements. From a space-based perspective, target detection performance should decrease when there is both increasing target eccentricity and increasing numbers of distractors falling to the center of the target. From an object-based perspective, however, a peripheral ring of elements can be grouped and selected together as a single object without the simultaneous selection of central elements. According to this view, ringbased cuing should make performance immune to increases in the relative eccentricities of targets and distractors.

Egly and Homa (1984; Experiment 3) used displays containing three concentric rings, one of which was precued. After cuing, two target elements (letters) were presented, one at fixation, and the other somewhere in the periphery, on one of the concentric rings. Central and peripheral elements were of the same size (cortical magnification was not controlled). Participants had to identify the central element and localize the peripheral one. Localization of a letter on the middle ring did not differ as a function of whether the inner or outer ring was precued. Egly and Homa interpreted this finding as evidence that attention was preallocated to the cued ring, and then, when the target was presented on an uncued ring, attention spread inward (when the target was presented to the center of the cued ring) or outward (when the target was peripheral to the cued ring) with equal efficiency. Their finding is incompatible with space-based theories that assume that the allocation of attention to the periphery necessarily involves the allocation of attention to the center. Egly and Homa concluded that a ring-shaped region of space can be presensitized by attention. Similar data have been reported by Eimer (1999).

Egly and Homa's (1984) results show that attention can be allocated to a ring in an empty field, but what happens when peripheral elements arranged in a ring are pitted against central elements favored by the central bias? When attention was allocated to the ring in the Egly and Homa study, there were no target and distractor elements present to compete for a response. When the probe appeared, it again did not have to compete with a central item for the same response. The conditions for eliciting the central bias were not met in this study, which therefore does not provide a strong test of the interaction between objectbased attention and the central bias in spatial selection.

Subsequently, Juola, Bouwhuis, Cooper, and Warner (1991) extended Egly and Homa's (1984) investigations by introducing multiple distractors. As in Wolfe et al. (1998), increasing numbers of distractors fell to the center of a target as it was moved further to the periphery. This ought to provide a better test of whether ring-based selection can override spatial selection of central elements.
However, despite the inclusion of distractors, performance did not decline with increasing target eccentricity. This was presumably because distractors differed from the target in a salient feature (see Treisman \& Gelade, 1980). In contrast, studies of the central spatial bias (e.g., Wolfe et al., 1998) have used distractor elements expressing target features and distinguished from targets only by conjunctions of features, making them into strong competitors for selection for report. In Juola et al. (1991), there is no evidence that distractors generated a central bias in selection.

No previous studies have demonstrated that objectbased selection of a peripheral ring can occur in the face of - and overrule - a central bias to select central stimuli for report. Such a result would be of theoretical importance, however, because it would suggest that object- and space-based attention can operate independently, counteracting one another in certain circumstances. We present this result in the current article. To generate space-based competition for selection, we followed Wolfe et al. (1998) and used multiple distractors that differed from targets in their conjunction of features. Then, to facilitate object-based cuing and selection, the display area over which the elements were presented was divided into three concentric rings, separated from each other by black regions of the display background. Target and distractor elements were always arranged on one or more of these rings (see Figures 1 and 2). As in Wolfe et al., distractors fell either all at the same eccentricity as targets (the one-ring arrangement; see Figure 1), or at multiple eccentricities (the multiple-ring arrangement; see Figure 2); the number of distractors falling closer to fixation than the target increased with the eccentricity of the target in the multiple-ring, but not in the one-ring, condition.

The ring containing the target was either predictable, when a $100 \%$ valid cue ${ }^{1}$ preceded the letters, or unpredictable, when no such cue occurred. With uncued displays, the central bias in spatial selection should dictate that RTs

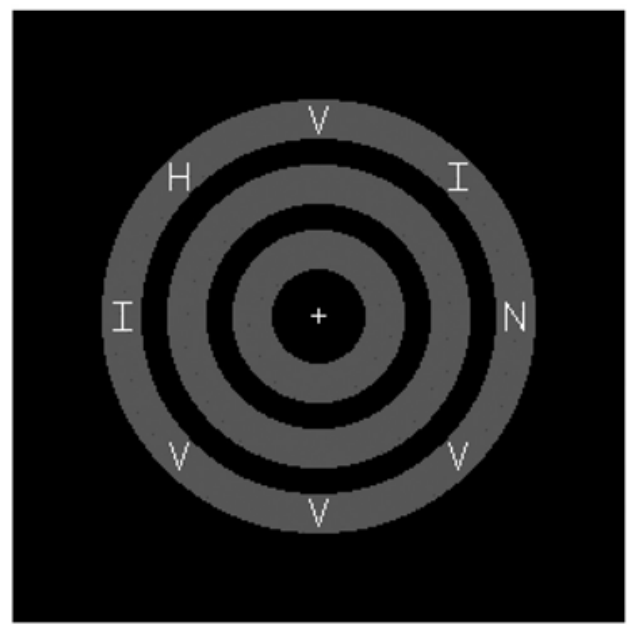

Figure 1. Experiment 1: Example display (in monochrome only) from the one-ring condition with the target presented on the outer ring. 


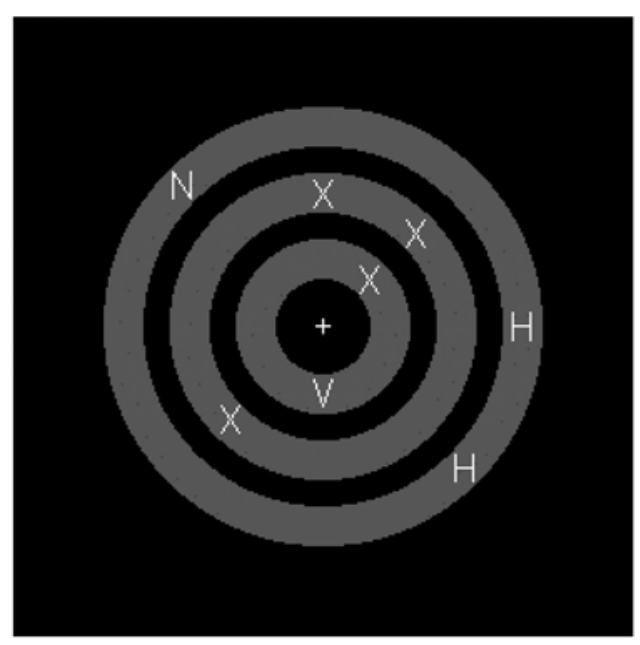

Figure 2. Experiment 1: Example display (in monochrome only) from the multiple-ring condition with the target presented on the outer ring.

are slower in multiple-ring than in one-ring displays, more so as target eccentricity increases (see Wolfe et al., 1998). We asked whether cuing attention to the target ring would abolish such a central bias. If so, then increasing the target's eccentricity should no longer lead to greater slowing of RTs in the multiple-ring, relative to the one-ring, condition.

\section{EXPERIMENT 1 \\ Conjunction Search on Uniform Gray Rings or Differently Colored Rings}

The target and distractor elements were white letters. Participants had to decide which of two target letters, "N" or "Z," was present among distractor letters that were made up of the same line elements as were the targets. This can be considered a form of conjunction search, since targets were distinguished only by their particular combination of features (see also Theeuwes, Kramer, \& Atchley, 1998). ${ }^{2}$ Target letters could occur in either the middle or outer of the three concentric rings presented as the backdrop for every display. In blocked conditions, target and distractor letters appeared either all on one ring (one-ring condition; Figure 1) or across all three rings (multiple-ring condition; Figure 2), and the ring on which the target letter appeared was either uncued or cued. The cue, when present, comprised the brightening of the entire ring at the target eccentricity $200 \mathrm{msec}$ prior to the exposure of all the letter elements. The cue was $100 \%$ valid, encouraging participants to attend to it endogenously. However, the fact that a short (200-msec) cue-stimulus onset asynchrony (SOA) was used makes it most likely that cuing operated exogenously (e.g., Cheal \& Lyon, 1991).

We also contrasted performance when the background ring-objects were either all the same shade of gray or differently colored (one red, one green, and one blue). Due to the phenomenon of chromostereopsis, differently colored rings can appear to occupy different planes in depth (Einthoven, 1885; Winn, Bradley, Strang, McGraw, \& Thibos, 1995). If any effects of cuing attention to a ring were due to attending to stimuli at different depths (a form of space-based attention, but in 3-D), then the cuing effects should be particularly striking with colored background rings (which segment more easily into different depths).

\section{Method}

Participants. Thirty-two participants (21 women and $11 \mathrm{men}$ ), with an average age of 20 years, took part in the experiment for course credit or money. They all had self-reported normal or corrected-to-normal vision. Sixteen performed the experiment with monochrome rings, and the remaining 16 with colored rings.

Apparatus. Stimuli were presented on a 17 -in. color monitor, driven by a Pentium 486 PC with a VESA graphics card running at a resolution of $640 \times 480 \times 256$. They were generated by a purpose-written Turbo-Pascal Version 7.0 program that recorded RTs and responses made via the computer keyboard. Stimulus luminance was controlled with the aid of a hand-held Minolta color meter (CS-100). The stimulus monitor was viewed from a fixed distance (see below) in a dimly lit room.

Stimuli. Stimuli were presented on a black background $\left(0 \mathrm{~cd} / \mathrm{m}^{2}\right)$. On every trial, a white $\left(80 \mathrm{~cd} / \mathrm{m}^{2}\right)$ fixation cross $\left(0.5^{\circ}\right.$ high and wide $)$ was presented at the center of three nested concentric rings. The rings were $1^{\circ}$ thick and were separated by bands of black background of the same thickness. The radius of the rings was $2^{\circ} \pm 0.5^{\circ}$ for the inner ring, $4^{\circ} \pm 0.5^{\circ}$ for the middle ring, and $6^{\circ} \pm 0.5^{\circ}$ for the outer ring. The rings were either all a uniform shade of gray, with a luminance of $5 \mathrm{~cd} / \mathrm{m}^{2}$, or differently colored, one red, one green, and one blue. The colored rings were approximately isoluminant with the gray rings. The assignment of red, green, and blue to the inner, middle, and outer rings was unpredictable on any trial but was controlled across the experiment so that no ring was more likely than the others to carry any one color.

Distributed over one or more (see below) of the rings were eight white letters $\left(80 \mathrm{~cd} / \mathrm{m}^{2}\right)$, measuring $0.8^{\circ}$ high $\times 0.5^{\circ}$ wide. The target letter was " $N$ " half the time and " $Z$ " half the time, and distractor letters were randomly chosen with replacement from a set of letters made up of "H," "I," "X," and "V." The distribution of the letters varied across one- and multiple-ring conditions. In the one-ring condition, all eight letters were arranged on one ring, either the middle ring or the outer ring. In this case, the letters were equally spaced around the particular ring, always occurring along the four cardinal directions and the four diagonals. In the multiple-ring condition, the eight letters were distributed across all three rings, with at least two, but no more than three, letters occurring on any one ring. The target letter always occurred on either the middle or the outer ring, together with two other letters. The positions of the two or three letters within each ring were randomly chosen from the four cardinal and four diagonal positions.

The ring on which the target letter occurred was unpredictable for the uncued condition, and on any one trial was equally likely to be the middle or outer ring. For the cued conditions, however, after the presentation of the rings but before the presentation of the letters (timings are given below), the entire surface of the gray or colored ring that would contain the target letter brightened by approximately $2 \mathrm{~cd} / \mathrm{m}^{2}$.

Procedure. The order of events on a cued trial was as follows: The three rings were presented for $1,500 \mathrm{msec}$; subsequently, the target ring brightened for $100 \mathrm{msec}$, before returning to its original brightness for $100 \mathrm{msec}$; finally, the letters were superimposed on the rings. On an uncued trial, the order was the same except that the target ring was not brightened, so that the rings were displayed for $1,700 \mathrm{msec}$ before the letters appeared. The letters remained in view 

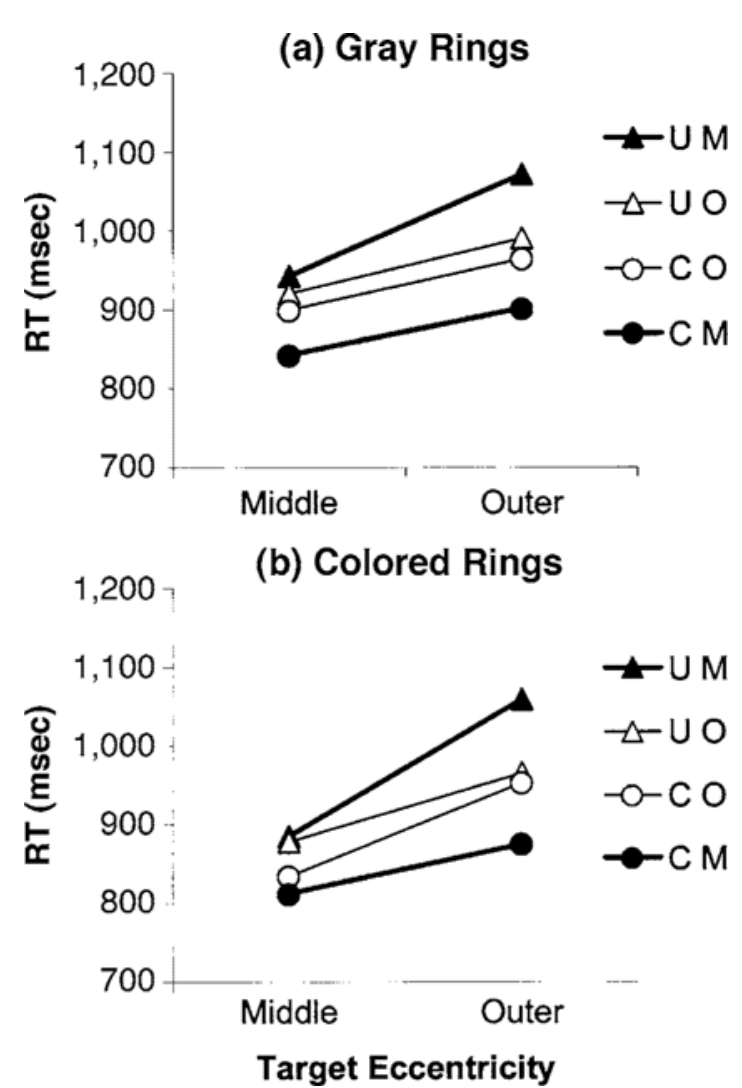

Figure 3. Experiment 1: Mean RTs as a function of target eccentricity (middle or outer ring) for uncued $(U)$ and cued $(C)$ trials with multiple-ring $(M)$ and one-ring $(O)$ arrangements of letters on (a) gray and (b) colored rings.

until the participant signaled whether the target was an "N" or a " $\mathrm{Z}$ " (by pressing the key corresponding to the target letter), or until $10 \mathrm{sec}$ had elapsed. Subsequently, the screen was blacked out for $500 \mathrm{msec}$ before the start of the next trial.

The participants were seated $45 \mathrm{~cm}$ from the screen. They were informed before any block of trials whether the letters would occur in one-ring or multiple-ring arrangements, and whether there would be cuing. If there was to be cuing, they were informed that it would be perfectly reliable. They were given a short block of practice trials before each block of 72 test trials, and they were asked to make their discrimination responses as quickly but as accurately as possible.

As a preliminary to Experiment 1, a separate group of 10 participants was shown displays containing concentric rings without any letters on them and asked to rate the apparent depth differences between the concentric rings. Each participant was shown both monochrome and differently colored concentric rings and asked to use a depth-difference scale that ran between 0 (no depth difference) and 7 (maximum depth difference). On average, the participants rated the depth differences between colored rings (4.3) to be greater than those between monochrome rings (2.2). This difference was significant on a two-tailed $t$ test $[t(9)=3.28, p<.01]$.

Design. There was one between-subjects factor: ring color (monochrome or colored). And there were three within-subjects factors: (1) cuing (target ring cued or uncued); (2) arrangement (letters arranged around the same ring, or distributed across the three possible rings); and (3) target eccentricity (target letter in the middle or outer ring). For each group of 16 participants, the cuing and arrangement factors were blocked, and the order of these blocks was counterbalanced across four subgroups of 4 participants: Two of these subgroups performed the cued conditions first, and the other two groups performed the uncued conditions first.

\section{Results}

Mean correct discrimination RTs and mean discrimination errors are shown as a function of target eccentricity in Figure 3 and Table 1, respectively, with performance broken down according to whether cuing was present or absent and whether letters appeared in one-ring or multiplering arrangements.

For both the RT and error data, a mixed-design analysis of variance (ANOVA) was conducted, with ring color as the between-subjects factor. The within-subjects factors were as follows: cuing (target ring either cued or uncued), arrangement (one-ring vs. multiple-ring), and target eccentricity (target in the middle or outer ring). For the error data, the only significant effect was that of arrangement $[F(1,30)=5.94, p<.021]$. For the RT data, there were significant main effects of cuing $[F(1,30)=27.14, p<.000]$ and target eccentricity $[F(1,30)=116.85, p<.000]$. There were significant two-way interactions between cuing and arrangement $[F(1,30)=14.85, p<.001]$ and between cuing and target eccentricity $[F(1,30)=10.59$, $p<.003]$. Finally, the three-way interaction between cuing, arrangement, and target eccentricity was also significant $[F(1,30)=12.90, p<.001]$. There was no main effect of ring color, and there were no interactions between ring color and any other factor or combination of factors.

The three-way interaction was broken down by analyzing the cued and uncued data separately. For uncued RTs, there were significant main effects of arrangement $[F(1,30)=$ $8.31, p<.007$; uncued RTs were slower to multiple-ring displays than to one-ring displays] and target eccentricity $[F(1,30)=120.12, p<.000]$; there was also a significant interaction between arrangement and target eccentricity $[F(1,30)=10.11, p<.003$; the slowing of multiple-ring compared with one-ring uncued RTs was greater at larger eccentricities; see Figure 3]. There were no interactions with ring color. For cued RTs, there were again main effects of arrangement $[F(1,30)=4.77, p<.037$; cued RTs were faster to multiple-ring displays than to one-ring displays; see Figure 3] and target eccentricity $[F(1,30)=$ $50.32, p<.000]$; however, cuing removed the interaction

Table 1

Experiment 1: Mean Percentage Errors as a Function of Target Eccentricity (Middle or Outer Ring)

for Uncued and Cued Trials With Multiple- and One-Ring Arrangements on Gray and Colored Rings

Target on Middle Ring Target on Outer Ring

\section{Gray Rings}

Uncued multiple-ring

Uncued one-ring

Cued one-ring

Cued multiple-ring

Colored Rings

Uncued multiple-ring

Uncued one-ring

Cued one-ring

Cued multiple-ring

3
2
3
3

3
2
2
4




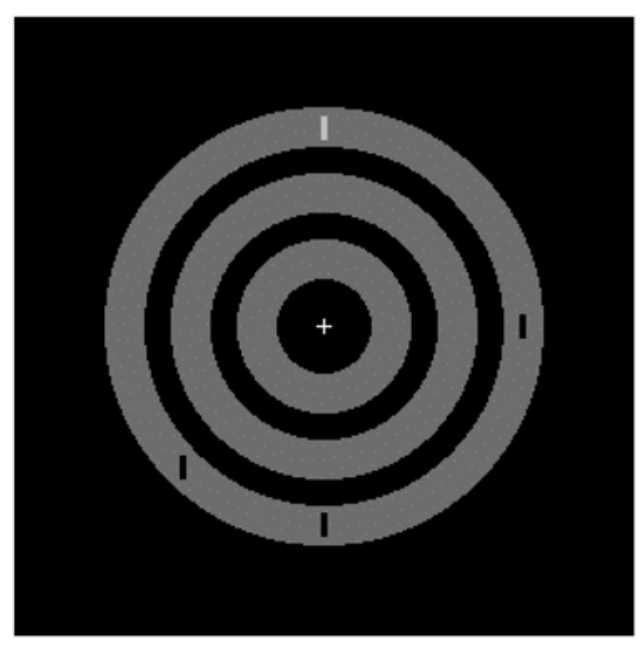

Figure 4. Experiment 2: Example display from the one-ring condition with the target presented on the outer ring.

between arrangement and target eccentricity found in the uncued data. There were no interactions with ring color.

\section{Discussion}

The uncued data essentially replicate those of Wolfe et al. (1998): There was an effect of target eccentricity in the multiple-ring condition that was greater than the effect in the one-ring condition. This effect is consistent with space-based attention being allocated to elements in proportion to their relative proximity to fixation.

The new result is that this effect of target eccentricity (in the multiple-ring condition compared with the onering condition) was eliminated when the target ring was cued. Apparently, attention could be allocated selectively to a peripheral ring, and performance then was not disrupted by more central distractors. Given that there was no effect of whether or not the background rings were colored, and that the rating study demonstrated that the colored rings segregated more strongly in depth, our result is inconsistent with the suggestion that selection was achieved by selecting a depth plane. Rather, the data are consistent with attention to individual ring-shaped objects overruling space-based attention. In fact, cuing led to a reversal of the absolute RT advantage that was present in the one-ring uncued condition: On cued trials, RTs tended to be faster in the multiple- than in the one-ring condition. We attribute this to fewer distractors competing for selection with the target within the cued ring in the multiple- as compared with the one-ring condition. This finding is again indicative of efficient selection of just the cued ring of elements.

In Experiment 2, we extended Experiment 1 by rearranging distractor elements so that, within the target ring, the target differed from distractors in a single feature. Feature search can operate in parallel within an attended area (Treisman \& Gelade, 1980). If there are no effects on search of the number of distractor elements within a ring, then we can argue that the ring was selected as a whole.
This rules out the possibility that participants attended to one part of a ring and then searched serially around the remaining parts of the ring.

\section{EXPERIMENT 2 \\ Conjunction Search Across Rings, Feature Search Within Rings}

In Experiment 2, the target differed from the distractors in the target ring by a simple feature difference-namely, contrast polarity (see Figure 4). The background rings were all a uniform shade of gray, and the target was a white line when the distractors in its ring were black lines, or a black line when the distractors in its ring were white lines. The task was to search for a "color" (contrast polarity) oddman-out within a ring. Search for an odd-man-out target can be spatially parallel and may even increase in efficiency with display set size (Sagi \& Julesz, 1987). To verify this, we conducted a pilot study with 4 participants to examine the impact of display set size (the number of elements within a ring) on target selection. The display set size was either four line elements per ring (as in the main experiment reported below) or six or eight line elements per ring. Performance was uncued, and the elements appeared in just one ring (inner or outer). The mean RTs and error percentages for the 4 participants are shown in Table 2 . For targets presented at both of the eccentricities used in the experiment, there was no evidence of any systematic increase in RTs as the number of elements in a ring increased. The error data support the RT data. It can be concluded that processing was spatially parallel within rings.

In the multiple-ring condition, we presented distractor elements in a second "nontarget" ring. These extra distractor elements shared their contrast polarity with the target element (black if it was black, or white if it was white) but had a different orientation from the target (vertical if it was horizontal, or horizontal if it was vertical; see Figure 5). This meant that, in multiple-ring displays, the target element was formally defined by a conjunction of contrast polarity and orientation features (either white and vertical on some trials, or black and horizontal on others) across the whole display. Prior studies have indicated that search for a conjunction target can be efficient when contrast polarity is one of the defining features (Theeuwes \& Kooi, 1994). This might be problematic if search became so efficient as to minimize any effects of distractor elements on

Table 2

Experiment 2: Mean Uncued Target-Present and TargetAbsent RTs and Error Percentages as a Function of Display Set Size (4, 6, or 8 Elements per Ring) for One-Ring Displays

\begin{tabular}{|c|c|c|c|c|c|c|}
\hline & \multicolumn{6}{|c|}{ Set Size } \\
\hline & \multicolumn{2}{|c|}{4} & \multicolumn{2}{|c|}{6} & \multicolumn{2}{|c|}{8} \\
\hline & $M$ & $\% \mathrm{E}$ & $M$ & $\% \mathrm{E}$ & $M$ & $\% \mathrm{E}$ \\
\hline Target present in inner ring & 605 & 0 & 584 & 0 & 599 & 2 \\
\hline Target present in outer ring & 616 & 0 & 589 & 4 & 567 & 2 \\
\hline Target absent & 606 & 2 & 626 & 1 & 593 & 4 \\
\hline
\end{tabular}




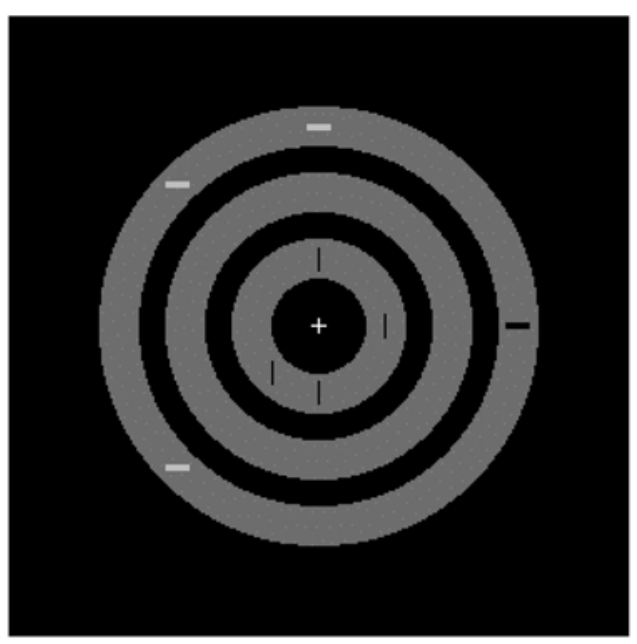

Figure 5. Experiment 2: Example display from the multiplering condition with the target presented on the outer ring.

search. However, we show that this was not the case in the uncued multiple-ring condition.

Three other changes in this study were as follows. First, the inner and outer rings were used as the backdrop for targets, rather than the middle and the outer rings, as previously. Targets in the inner ring fell within the anatomical fovea, whereas those falling on the outer ring fell well outside of it. By distributing display elements in this way, we attempted to maximize the opportunity for the central bias in spatial attention to affect performance. In order to control for the large changes in cortical magnification that accompany the move from extrafoveal to foveal vision, elements that fell on the inner ring were scaled down in size in comparison with those falling on the outer ring. We followed Carrasco and Frieder (1997) in using a magnification factor derived from the early behavioral work of Virsu and Rovamo (1979). There is more recent fMRI evidence (Engel, Glover, \& Wandell, 1997) that this factor constitutes a very conservative estimate of the degree of cortical magnification in the fovea.

The second change was to alter one-ring displays so that they contained only half as many elements as multiplering displays. This meant that there were the same number of elements in one-ring displays as in the target rings in multiple-ring displays. Now, when the target ring was cued, we could assess whether distractors outside the target ring exerted any impact on search, by comparing the multiple-ring data with the corresponding one-ring baseline.

Finally, the SOA between the presentation of the cue and the target and distractor stimuli could be $200 \mathrm{msec}$ (as in Experiment 1) or $600 \mathrm{msec}$. Efficient cuing after an interval of only $200 \mathrm{msec}$ is typical of exogenous cuing, as we found in Experiment 1. In contrast, pilot runs of Experiment 2 suggested that cuing with 200 -msec SOAs was only partially efficient. Objects can be cued endogenously as well as exogenously (see Abrams \& Law, 2000, and Duncan, 1984) and, if the effects of these two types of cuing can summate, then cuing should be improved with a 600msec SOA, long enough to support both types of cuing.

\section{Method}

Unless otherwise mentioned, the method was the same as in Experiment 1 .

Participants. Twelve participants ( 8 women and 4 men), with an average age of 27 years, took part in the experiment for course credit or money. They all had self-reported normal or corrected-to-normal vision.

Stimuli. The average radius of inner, middle, and outer rings was $1.5^{\circ}, 3.0^{\circ}$, and $4.5^{\circ}$, respectively, with a ring width of $0.75^{\circ}$. The brightness of the rings was $14 \mathrm{~cd} / \mathrm{m}^{2}$. They were arranged on a black $\left(0 \mathrm{~cd} / \mathrm{m}^{2}\right)$ background with a bright white $\left(80 \mathrm{~cd} / \mathrm{m}^{2}\right)$ fixation cross at the center. Display elements were black $\left(0 \mathrm{~cd} / \mathrm{m}^{2}\right)$ or white $\left(28 \mathrm{~cd} / \mathrm{m}^{2}\right)$, horizontal- or vertical-line elements, presented on the inner and/or the outer of three gray concentric rings. No elements were presented on the middle ring. Elements subtended $0.5^{\circ} \times 0.05^{\circ}$ when presented on the inner ring, but $0.5^{\circ} \times 0.15^{\circ}$ when presented on the outer ring, to control for cortical magnification. One-ring displays contained four elements, all in either the inner or the outer ring. Multiple-ring displays contained eight elements, four in each of the inner and outer rings. Elements were arranged so that their midpoints occurred at the average radius of their ring, along any one of the four cardinal or four diagonal directions. Within each occupied ring, the four elements were allocated randomly to four of the eight possible locations. Example one- and multiple-ring displays are shown in Figures 4 and 5, respectively.

Line elements could be of four types: white horizontal or black vertical (distractor) elements, and white vertical or black horizontal (target) elements. The two types of distractor element never occurred together within the same ring. Thus, one-ring displays contained only one distractor type, whereas multiple-ring displays contained both types, one type in the inner ring, and the other type in the outer ring (half the time, white horizontal elements in the inner ring and black vertical elements in the outer ring; the remaining time, black vertical elements in the inner ring and white horizontal elements in the outer ring). When a target occurred (on half the trials), it differed from the distractor elements in its ring in contrast polarity but not orientation. Consequently, the target was defined as a contrast-polarity odd-man-out within its ring. The target appeared with equal likelihood across trials on the inner and outer rings. On cued trials, the ring on which the target was to appear was brightened by $14 \mathrm{~cd} / \mathrm{m}^{2}$ for $100 \mathrm{msec}$. On uncued trials, the target occurred unpredictably on either the inner or the outer ring.

Procedure. The participants were asked to keep their eyes on the white fixation cross throughout the trial, and they were told that looking to one part of a ring would not convey any advantage. Their task was to judge whether or not there was an "odd-colored" element in the cued ring on cued trials, or in either the inner or the outer ring on uncued trials, and to make speeded "present" or "absent" responses accordingly (with the index fingers of their dominant and nondominant hands, respectively). The order of events on cued trials was as follows: (1) fixation cross and equiluminant rings displayed for $900 \mathrm{msec}$; (2) target ring brightened for $100 \mathrm{msec}$; (3) equiluminant rings displayed again for either 500 (600-msec SOA) or 100 (200-msec SOA) msec; and (4) line elements presented on rings. On uncued trials, the equiluminant rings were displayed for $1,500 \mathrm{msec}$, and then the line elements were presented on the rings. On both cued and uncued trials, line elements remained visible until a response was made or $10 \mathrm{sec}$ had elapsed, and then the display was blacked out for $500 \mathrm{msec}$ before the start of the next trial.

The participants were seated $55 \mathrm{~cm}$ from the screen. They were informed before any block of trials whether line elements would occur in one-ring or multiple-ring arrangements and whether there would be cuing. They were given a short block of practice trials before each block of 80 test trials. 

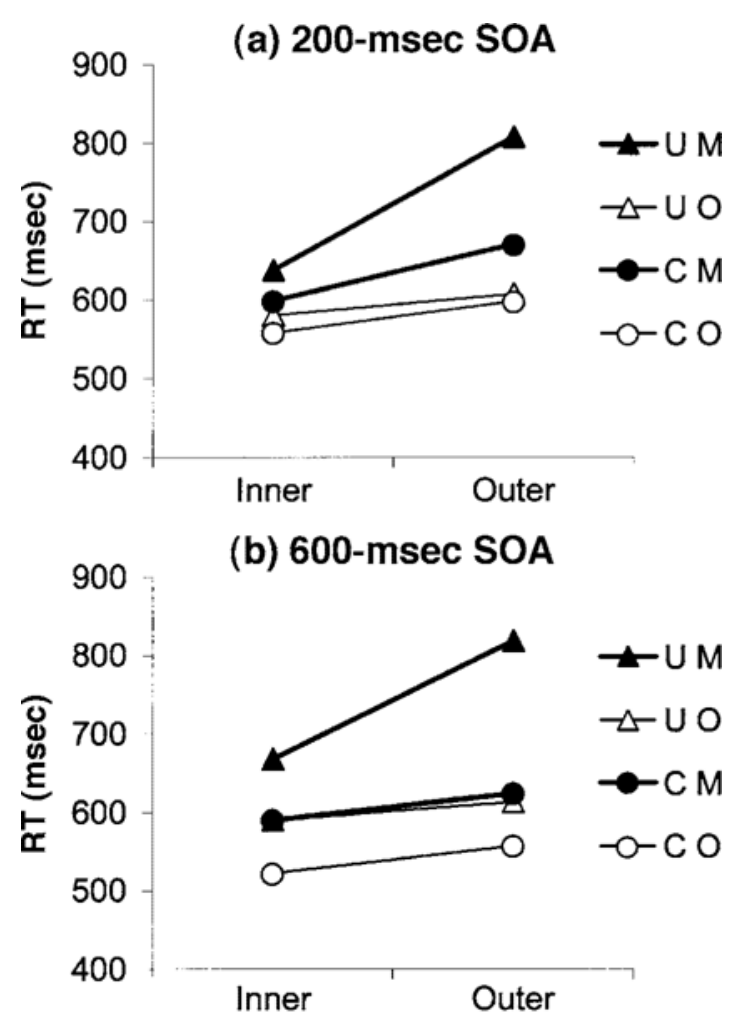

Target Eccentricity

Figure 6. Experiment 2: Mean target-present RTs as a function of target eccentricity (inner or outer ring) for uncued $(U)$ and cued $(C)$ trials with multiple-ring $(M)$ and one-ring $(O)$ arrangements of lines for (a) 200-msec cue-stimulus SOAs and (b) 600msec cue-stimulus SOAs.

Design. There were three within-subjects factors: cuing (target ring cued or uncued), arrangement (one-ring or multiple-ring) and target eccentricity (target element in inner or outer ring). Cuing and arrangement were blocked, ${ }^{3}$ and the order of these blocks was counterbalanced across four subgroups of 3 participants: Two of these subgroups performed the cued conditions first and the other two performed the uncued conditions first. Within the cued part of the experiment, the participants always performed both arrangement blocks at a 200-msec SOA and then repeated them in the same order at a 600-msec SOA. Thus, cue-stimulus SOA was confounded with practice, although there were always practice trials at the beginning of each block.

\section{Results}

Mean correct target-detection RTs and mean targetdetection errors (misses) are shown as a function of target eccentricity in Figures $6 \mathrm{a}$ and $7 \mathrm{a}$, respectively. The cued data in Figures $6 \mathrm{a}$ and $7 \mathrm{a}$ are from the 200-msec SOA, whereas the cued data in Figures $6 \mathrm{~b}$ and $7 \mathrm{~b}$ are from the $600-\mathrm{msec}$ SOA. One of the participants did not adequately complete the cued conditions for the 200-msec SOA, so Figures $6 \mathrm{a}$ and $7 \mathrm{a}$ show the group means for the remaining 11 participants. In each figure, performance is broken down according to whether cuing was present or absent and whether line elements were confined to one ring (one-ring arrangement) or distributed across different rings (multiplering arrangement).
Three-way repeated-measures ANOVAs were conducted on both the RT and the error data from target-present trials. The factors were cuing, arrangement, and target eccentricity. The uncued performance was compared either with the 200-msec SOA cued performance (11-participant comparison) or with the 600 -msec SOA cued performance (12-participant comparison).

RT analyses: 200-msec SOA. There were significant main effects of cuing $[F(1,10)=7.95, p<.018]$, arrangement $[F(1,10)=31.22, p<.000]$, and target eccentricity $[F(1,10)=22.52, p<.001]$. There were significant two-way interactions between cuing and arrangement $[F(1,10)=9.22, p<.013]$, cuing and target eccentricity $[F(1,10)=14.40, p<.004]$, and arrangement and target eccentricity $[F(1,10)=25.46, p<.001]$. There was a significant three-way interaction between cuing, arrangement, and target eccentricity $[F(1,10)=15.83, p<.003]$. The effects of eccentricity were stronger in the uncued than in the cued condition for the multiple- relative to the one-ring condition. Nevertheless, there were some effects of eccentricity even on cued trials. On cued trials, RTs were slower to multiple-ring displays than to one-ring displays $[F(1,10)=22.09, p<.001]$, and this effect increased at larger target eccentricities $[F(1,10)=7.03, p<.024]$. On
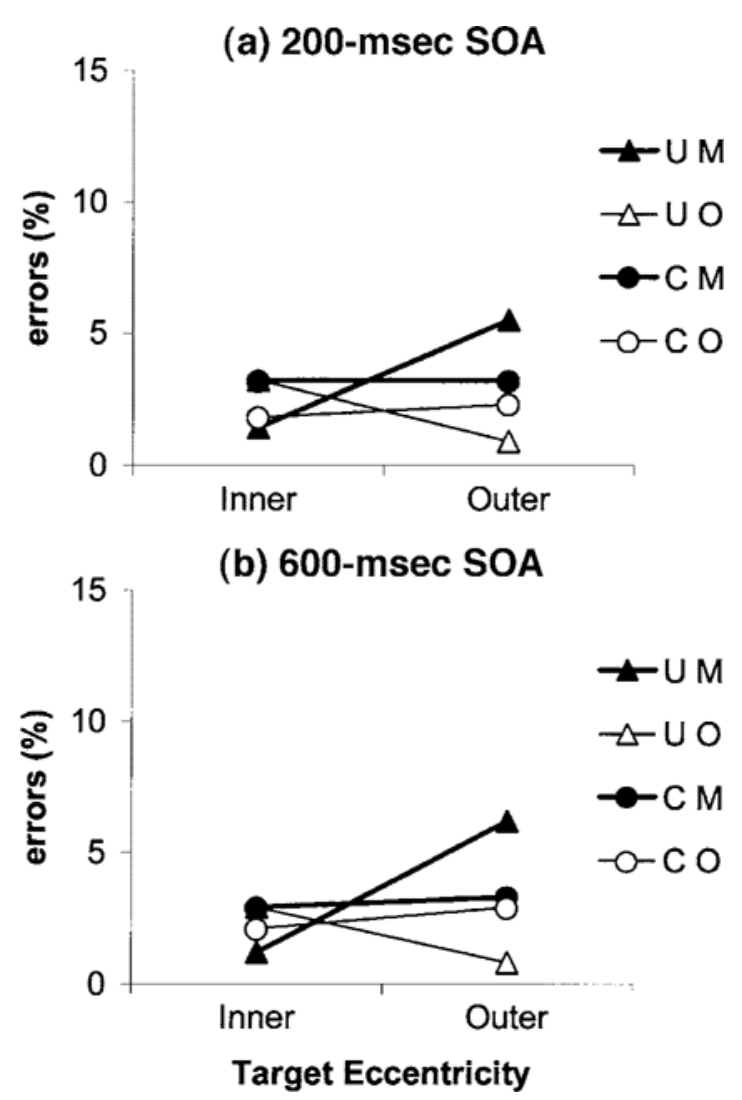

Figure 7. Experiment 2: Mean target-present error percentages as a function of target eccentricity (inner or outer ring) for uncued $(U)$ and cued $(C)$ trials with multiple-ring $(M)$ and onering $(\mathrm{O})$ arrangements of lines for (a) 200-msec cue-stimulus SOAs and (b) 600-msec cue-stimulus SOAs. 
uncued trials, RTs were slower to multiple-ring displays than to one-ring displays $[F(1,10)=24.19, p<.001]$, and this effect, too, increased as target eccentricity increased $[F(1,10)=24.59, p<.001]$.

Error analyses: 200-msec SOA. There were no significant effects, but the three-way interaction between cuing, arrangement, and target eccentricity $[F(1,10)=3.27, p<$ .1] approached significance. When the cued and uncued data were analyzed separately, the number of errors to multiple-ring and one-ring arrangements interacted with target eccentricity on uncued trials $[F(1,10)=4.67, p<$ $.056]$ but not on cued trials $[F(1,10)=0.07, p<.796]$. Inspection of Figure 7A indicates that the error data largely followed the RT data.

RT analyses: 600-msec SOA. There were significant main effects of cuing $[F(1,11)=91.72, p<.000]$, arrangement $[F(1,11)=50.40, p<.000]$, and target eccentricity $[F(1,11)=14.59, p<.003]$. There were significant two-way interactions between cuing and arrangement $[F(1,11)=4.59, p<.055]$, cuing and target eccentricity $[F(1,11)=9.38, p<.011]$, and arrangement and target eccentricity $[F(1,11)=11.68, p<.006]$. There was a significant three-way interaction between cuing, arrangement, and target eccentricity $[F(1,11)=14.31, p<.003]$. When the cued and uncued data were analyzed separately, uncued RTs were slower to multiple-ring displays than to one-ring displays $[F(1,11)=27.53, p<.000]$, and this effect interacted with target eccentricity $[F(1,11)=18.42$, $p<.001]$. In contrast, although cued RTs were slower to multiple-ring displays than to one-ring displays $[F(1,11)=$ $15.50, p<.002]$, there was no interaction with target eccentricity.

Error analyses: 600-msec SOA. There was a significant main effect of target eccentricity $[F(1,11)=5.85$, $p<.034]$. There was also a significant three-way interaction between cuing, arrangement, and target eccentricity $[F(1,11)=14.14, p<.003]$. The number of errors to multiple-ring and one-ring displays interacted with target eccentricity on uncued trials $[F(1,11)=6.47, p<.027]$ but not on cued trials $[F(1,11)=0.02, p<.889]$.

\section{Discussion}

There was a strong effect of eccentricity on target detection in the uncued condition with multiple rings of elements. In contrast, there was no effect of eccentricity in the onering baseline (indeed, errors tended to decrease to more eccentric targets in the one-ring baseline). Thus, the effect of target eccentricity with multiple rings can be attributed to an attentional rather than a visual effect - namely, the bias to favor elements at more foveal locations over those at more peripheral locations (Wolfe et al., 1998). Despite the strength of this bias, cuing attention to the target ring significantly reduced the eccentricity effect in the multiplering condition for the 200 -msec SOA and completely eliminated it for the 600-msec SOA. Apparently, objectbased cuing was able to overcome the effects of a spatial bias in visual selection, even when extrafoveal elements were put into competition with foveal ones (cf. Experi- ment 1). This cuing effect arose under conditions in which the target was selected in a spatially parallel manner (see Table 2 and the error data that are incompatible with eye movements) and thus provides strong evidence for objectbased selection in vision.

One other notable result was the increase in RTs for multiple-ring over one-ring trials, even when the target ring was successfully cued (see Figure 6B). The fact that this increase did not interact with target eccentricity (for 600-msec SOAs) demonstrates that it was not due to spacebased biases in selection. Instead, the result may reflect a form of "filtering cost" (e.g., Kahneman, Treisman, \& Burkell, 1983). Perhaps onsetting distractors lessened the efficiency with which the pop-out target was selected by competing with it for resources (see Joseph, Chun, \& Nakayama, 1997, for evidence that competition for attentional resources can affect even simple feature search).

Our data raise the question of why a $600-\mathrm{msec}$ SOA was necessary to produce perfect cuing in Experiment 2, whereas a 200-msec SOA sufficed in Experiment 1. As has already been stated, 200-msec SOAs are typical of exogenous, bottom-up cuing, whereas longer SOAs may support the involvement of endogenous, top-down processes. Why might it have been necessary to supplement exogenous orienting with endogenous orienting in Experiment 2? Eimer (1999) has compared the ERPs that result from cuing participants to attend to contiguous quadrants as compared with noncontiguous rings and concluded that qualitatively different mechanisms of attention (perhaps endogenous attention) may be required in order to select a ring-shaped object. But why, then, should the data differ in Experiment 1? A possibility is that competition from central elements may be stronger when they fall within the anatomical fovea (as in Experiment 2, but not in Experiment 1). In this case, top-down, endogenous processes may be required to decrease the influence of central elements, perhaps through active processes of inhibition (Lavie, 1995, 2000).

\section{SUMMARY AND CONCLUSIONS}

In two experiments, we demonstrated that a spatial bias favoring central over peripheral stimulus elements can be eliminated by cuing participants to attend selectively to an outer ring containing the peripheral elements. In Experiment 1 , the cuing effect was demonstrated with a standard conjunction search task. In Experiment 2, it occurred under conditions in which each ring of elements was searched in a spatially parallel way, ruling out the suggestion that participants attended to a part of the cued ring and then moved a spatial spotlight of attention around the ring until a target was detected. Another space-based strategy, attending to rings in different depth planes, is also unlikely, since we found no enhancement of performance with colored rings that produced stronger depth segregation (Experiment 1). Finally, eye movements were unlikely to have led to more efficient search in the cued relative to the uncued condition, given that we cued peripheral rings with radii of at 
least $4.5^{\circ}$ and participants had no idea of the location of the target element within these rings. Making an eye movement to one of the eight potential target locations within a cued ring should have disrupted, rather than facilitated, performance when targets fell across such disparate areas of visual field; misses should have increased, particularly when the peripheral ring was cued, yet there is no evidence for this in the error data.

One difference between the two experiments was that a longer cue-target SOA was needed in Experiment 2 in order to eliminate the effects of eccentricity on performance. This suggests the involvement of endogenous cuing, which might have been necessary in Experiment 2 (but not in Experiment 1) in order to counteract the influence of foveal elements, even with cortical magnification controlled for (Jüttner \& Rentschler, 2000). Thus, it might have been necessary for foveal elements to be actively suppressed for ring-based selection of the extrafoveal elements to occur. Suppression might also have been particularly necessary in Experiment 2 because the displays constituted lower perceptual loads than those in Experiment 1 ; there can be greater processing of distractors under low-load conditions (see Lavie, 1995, 2000).

However, although interesting, the effects of SOA are tangential to our main interest, which concerns the finding that a peripheral ring of elements can be selected in parallel and at the expense of central elements that otherwise win the competition for selection. We conclude that it is possible to select spatially noncontiguous ring-shaped objects - where ring-shaped objects are defined either by a grouped ring of locations or by a spatially invariant representation - and, in doing this, to overrule the central bias in space-based selection. These behavioral data are consistent with the idea that object-based selection operates independently of the central bias in space-based selection.

\section{REFERENCES}

Abrams, R. A., \& Law, M. B. (2000). Object-based visual attention with endogenous orienting. Perception \& Psychophysics, 62, 818-833.

Bundesen, C. (1990). A theory of visual attention. Psychological Review, 97, 523-547.

Carrasco, M., Evert, D. L., Chang, I., \& Katz, S. M. (1995). The eccentricity effect: Target eccentricity affects performance on conjunction searches. Perception \& Psychophysics, 57, 1241-1261.

Carrasco, M., \& Frieder, K. S. (1997). Cortical magnification neutralizes the eccentricity effect in visual search. Vision Research, 37 , 63-82.

CHEAL, M., \& LyON, D. R. (1991). Central and peripheral precuing of forced-choice discrimination. Quarterly Journal of Experimental Psychology, 43A, 859-880.

DUNCAN, J. (1984). Selective attention and the organization of visual information. Journal of Experimental Psychology: General, 113, 501-517.

Egly, R., \& Homa, D. (1984). Sensitization of the visual field. Journal of Experimental Psychology: Human Perception \& Performance, $\underline{\mathbf{1 0}}_{2}$ 778-793.

EIMER, M. (1999). Attending to quadrants and ring-shaped regions: ERP effects of visual attention in different spatial selection tasks. Psychophysiology, 36, 491-503.

EINTHOVEN, W. (1885). Stereoscopie durch Farbendifferenz [Stereo- scopy through color differences]. Albrecht von Graefes Archiv für Ophthalmologie, 31, 211-238.

Engel, S. A., Glover, G. H., \& Wandell, B.A. (1997). Retinotopic organization in human visual cortex and the spatial precision of functional MRI. Cerebral Cortex, 7, 181-192.

Joseph, J. S., Chun, M. M., \& NaKayama, K. (1997). Attentional requirements in a "preattentive" feature search task. Nature, 387, 805-807.

Juola, J. F., Bouwhuis, D. G., CoOper, E. E., \& Warner, C. B. (1991). Control of attention around the fovea. Journal of Experimental Psychology: Human Perception \& Performance, 17, 125-141.

JÜTTNER, M., \& RENTSCHLER, I. (2000). Scale-invariant superiority of foveal vision in perceptual categorisation. European Journal of Neuroscience, 12, 353-359.

Kahneman, D., Treisman, A., \& Burkell, J. (1983). The cost of visual filtering. Journal of Experimental Psychology: Human Perception \& Performance, 9, 510-522.

LABERgE, D., \& BROWN, V. (1989). Theory of attentional operations in shape identification. Psychological Review, 96, 101-124.

Lavie, N. (1995). Perceptual load as a necessary condition for selective attention. Journal of Experimental Psychology: Human Perception \& Performance, 21, 451-468.

LAVIE, N. (2000). Selective attention and cognitive control: Dissociating attentional functions through different types of load. In S. Monsell \& J. Driver (Eds.), Control of cognitive processes: Attention and performance XVIII (pp. 175-194). Cambridge, MA: MIT Press.

SAGI, D., \& JulEsz, B. (1987). Short-range limitation on detection of feature differences. Spatial Vision, 2, 39-49.

Theeuwes, J., \& KooI, F. L. (1994). Parallel search for a conjunction of contrast polarity and shape. Vision Research, 34, 3013-3016.

Theeuwes, J., Kramer, A. F., \& AtChley, P. (1998). Visual marking of old objects. Psychonomic Bulletin \& Review, 5, 130-134.

Treisman, A., \& Gelade, G. (1980). A feature integration theory of attention. Cognitive Psychology, 12, 97-136.

VECERA, S. P., \& FARAH, M. (1994). Does visual attention select objects or locations? Journal of Experimental Psychology: General, 123, 146-160.

VIRSU, V., \& Rovamo, J. (1979). Visual resolution, contrast sensitivity, and the cortical magnification factor. Experimental Brain Research, 37, 475-494.

WinN, B., Bradley, A., Strang, N. C., McGraw, P. V., \& Thibos, L. N. (1995). Reversals of the colour-depth illusion explained by ocular chromatic aberration. Vision Research, 35, 2675-2684.

Wolfe J. M., O'Neill, P., \& BennetT, S. C. (1998). Why are there eccentricity effects in visual search? Visual and attentional hypotheses. Perception \& Psychophysics, 60, 140-156.

\section{NOTES}

1. Previous studies have all used cues that are occasionally unreliable. They have then contrasted the differences between validly and invalidly cued trials to infer the spatial distribution of attention. With our design, however, the inclusion of invalid trials becomes unnecessary.

2. A form conjunction search was used in Experiment 1 (rather than a color-form conjunction task like that employed by Wolfe et al., 1998) for compatibility with the differently colored rings we used as a backdrop for target and distractor elements. If we had used a color-form conjunction task, differently colored elements would have appeared against contrasting color backgrounds, which would have generated unwanted variance in the data.

3. The cuing factor was blocked to encourage participants to use the cue as much as possible. The arrangement factor was blocked to prevent participants from missing peripheral targets in multiple-ring displays. However, we have replicated the findings with the conditions randomly intermixed.

(Manuscript received June 18, 2002; revision accepted for publication October 5, 2003.) 